September, 1993

Report LPQTH-93/18

\title{
PERSISTENT CURRENTS IN 1D DISORDERED RINGS OF INTERACTING ELECTRONS
}

\author{
G.BOUZERAR日, D. POILBLANC因 \\ and \\ G. MONTAMBAUX田 \\ Groupe de Physique Théorique, \\ Laboratoire de Physique Quantique, \\ Université Paul Sabatier, \\ 31062 Toulouse, France
}

(Received )

\begin{abstract}
We calculate the persistent current of 1D rings of spinless fermions with shortrange interactions on a lattice with up to 20 sites, and in the presence of disorder, for various band fillings. We find that both disorder and interactions always decrease the persistent current by localizing the electrons. Away from half-filling, the interaction has a much stronger influence in the presence of disorder than in the pure case.
\end{abstract}

PACS numbers: 72.10.-d, 71.27.+a, 72.15.Rn

Typeset Using REVTEX 
The recent discovery of persistent currents in mesoscopic rings has addressed new interesting questions on the thermodynamics of these systems. Although such an effect was predicted for a long time, the unexpectedly large amplitude of the measured currents lead to important interrogations. Among them, the role of e-e interactions is still unclear. It has been proposed [1] that the interactions contribute to the average current which is measured in a many rings experiments [2]. On the other hand, the importance of the choice of the statistical ensemble to calculate average values has also been stressed [3]. Although the first explanation, based on a perturbative calculation both in interaction and disorder, seems to give a quantitative estimate closer to the experiment, it is still too small by one order of magnitude and the interaction parameter used in the theory is not well known.

In addition, for a single ring experiment [4], the magnitude of the measured current is also not understood and, up to now, perturbation theory has failed to explain an enhancement of the current [5]. It is only when disorder is weaker that experiment and theory seem to agree [6], even for non interacting electrons.

At the moment, the role of the interactions in disordered systems is still unclear and the subject is vastly open. It has been recently proposed that, in the presence of interactions, the current should be larger than for free electrons, the effect of the interactions being to counteract the disorder effect [7].

The aim of this paper is to describe the interplay between the interactions and the disorder on the persistent currents in $1 \mathrm{D}$ rings. We choose a model of spinless fermions with short range interactions on a lattice. Our main result is that a repulsive interaction always decreases the amplitude of the current. It is well known that the $1 \mathrm{D}$ description is certainly not the most appropriate one to describe quantitatively experiments which are performed in rings with finite width, in the diffusive regime. But our hope is to find numerical results which may give indications for a more real situation.

We describe a chain of $1 \mathrm{D}$ spinless fermions in the presence of disorder with the following Hamiltonian :

$$
\mathcal{H}=-t / 2 \sum_{\mathbf{i}}\left(\exp (2 i \pi \Phi / N) c_{\mathbf{i}}^{\dagger} c_{\mathbf{i}+\mathbf{1}}+\text { h.c. }\right)+V \sum_{\mathbf{i}} n_{\mathbf{i}} n_{\mathbf{i}+\mathbf{1}}+\sum_{\mathbf{i}} w_{\mathbf{i}} n_{\mathbf{i}}
$$

where $w_{\mathbf{i}}$ are on-site energies and are chosen randomly between $-\mathrm{W} / 2$ and $\mathrm{W} / 2$ and $\mathrm{V}$ is 
the nearest neighbour Coulomb repulsion. In the following we will take $t=1, \Phi$ is the total magnetic flux through the ring (measured in units of flux quantum $\Phi_{0}=h / e$ ) and $\mathrm{N}$ is the number of sites. (We use the conventional notation for the amplitude of disorder $\mathrm{W}$ and for the nearest neighbors interaction $\mathrm{V}$. These notations are opposite to those used in ref. [7]).

Let us first recall some physical properties of this hamiltonian without disorder, i.e. $\mathrm{W}=0$. In one dimension, for repulsive interaction, a metal-insulator transistion occurs at half-filling due to the existence of umklapp processes. However away from half-filling, the umklapp processes become irrelevant and the system is expected to be metallic [8]. For Hamiltonian (0.1), the metal-insulator (Mott) transition occurs at $V=1$. The system is insulator for $V>1$ and metallic for $0 \leq V<1$.

This transition can also be described in the spin picture [8]. In the case $W=0$, the model is integrable and is formally equivalent to an anisotropic spin model, as obtained by a standard Wigner-Jordan transformation. By this way the new Hamiltonian reads:

$$
\mathcal{H}_{X X Z}=-t / 2 \sum_{\mathbf{i}}\left(\exp (2 i \pi \Phi / N) S_{\mathbf{i}}^{+} S_{\mathbf{i}+\mathbf{1}}^{-}+\text {h.c. }\right)+V\left[N / 4+\sum_{\mathbf{i}} S_{\mathbf{i}}^{z} S_{\mathbf{i}+\mathbf{1}}^{z}\right]
$$

For the XXZ model, $\mathrm{V}=1$ corresponds to a transition from an XY-model $(V<1)$ to an Ising model $(V>1) . \quad V=1$ corresponds to an isotropic Heisenberg system. Note that a spin gap opens up for $V>1$. It corresponds to the gap in the charge excitations for Hamiltonian (0.1) characteristic of the insulator. With disorder, $W \neq 0$, we must add in (0.2), the following term

$$
\mathcal{H}_{\text {random }}=\sum_{\mathbf{i}} w_{\mathbf{i}}\left(S_{\mathbf{i}}^{z}-1 / 2\right)
$$

This term describes the interaction of the local spins with random magnetic fields.

We now turn to the numerical calculation of the ground state energy as a function of the total magnetic flux $E(\Phi)$ which is the first step of our work. The ground state energy is obtained from a standard Lanczos algorithm [9]. First of all, let us briefly describe some technical aspects of the method. The calculation is limited to relatively small system sizes $\mathrm{N}$, since (i) the Hilbert space dimension grows exponentially fast with $\mathrm{N}$, (ii) we have to average over many realizations of the disorder, (because of statistical fluctuations) and (iii) the disorder breaks the translation symmetry. The system sizes we were interested in, vary 
from 6 to 20 sites and we have chosen to consider two different cases: half and quarter fillings. Let us remind that the Lanczos method consists in the construction of a tridiagonal matrix by applying iteratively the Hamiltonian on an initial random vector. By this way a basis of normalized vectors $\Psi_{n}$ is defined as well as a set of values $e_{n}$ and $b_{n}$ given by the relationship, $\mathcal{H} \Psi_{n}=b_{n-1} \Psi_{n-1}+e_{n} \Psi_{n}+b_{n+1} \Psi_{n+1}$. Hence we construct by iteration a tridiagonal Hamiltonian matrix expressed in the $\Psi_{n}$ basis that we diagonalize to obtain the spectrum of the eigenvalues. This kind of process is rapidly converging.

Let us, now, consider the calculation of the persistent current in such rings threaded by a total flux $\Phi$. As usual the current is defined by

$$
I(\Phi)=-\frac{1}{2 \pi} \frac{\partial E(\Phi)}{\partial \Phi},
$$

where $E(\Phi)$ is calculated by exact diagonalization of the Hamiltonian. As well known, the flux can be gauged out from the Hamiltonian so that the presence of an Aharonov-Bohm flux through a ring is analogous to a twist in the boundary conditions $\Psi(x+N)=\Psi(x) e^{2 i \pi \Phi}$. The spectrum and the persistent current have the flux periodicity of one.

In figs.1,2, $I(\Phi)$ is plotted versus $\Phi$ for a 16 site ring at electron density $\langle n\rangle=0.5$ and $\langle n\rangle=0.25$ in (a) and (b) respectively, for different values of the disorder and the interaction. Fig. 1 corresponds to the 'ordered' interacting case $W=0$ and disorder $W=0.5$ is introduced in fig.2. On fig.1, a discontinuity of $I(\Phi)$ appears at $\Phi=0$, for zero disorder $(W=0)$. Indeed, in the absence of disorder, translation symmetry is preserved and total momentum is a good quantum number. As a function of $\Phi$, a crossing occurs between two lowest energy levels with different momenta. This crossing occurs at $\Phi=0$ for an even number of electrons or at $\Phi=0.5$ for an odd number. This can be easily understood in the non interacting case where $E(\Phi)=-\sum_{(n \in \mathcal{E}(n))} \cos \left(k_{n}+2 \pi \Phi / N\right)$ and the subset of the electron momenta $k_{n}=2 \pi n / N$ is chosen in order to minimize the total energy. Since translation invariance is preserved in the presence of interactions, the discontinuity still exists for finite $V$. When disorder is introduced $(W \neq 0)$ in fig.2, the scattering potential lifts the degeneracy at the crossing point and hence leads to a continuous variation of the current. In fig.1a (half-filling) we clearly observe the effect of the Mott transition on the currents: we notice that for $V<1$, I is slowly varying with $\mathrm{V}$, but when $V>1$ a drop of the current 
appears. However, away from half-filling (see fig.1b), the current is not affected for moderate interactions. At $W=0$ and away from half-filling, the system is always metallic.

In figs.2a and b, the influence of the repulsive interaction is shown for a fixed impurity potential of magnitude $W=0.5$ and for the same parameters as in figs. $1(\mathrm{~N}=16,<n>=0.5$ and $\langle n\rangle=0.25$ ). Clearly, in the half-filled case (fig.2a), the repulsion tends to suppress the current even further. This is reminicent of the Mott localization which occurs in the pure system. With increasing $\mathrm{W}, I(\Phi)$ decreases as expected due to a stronger localization by the impurity potential. Such an effect has also been found in a ring of spinless fermions with long-range interactions 11$]$.

More interesting is the effect of the interaction away from half-filling where no localization is expected in the absence of disorder. As seen previously in fig.1b the effect of the interaction for $W=0$ is extremely weak, because of the absence of Umklapp scattering. However, it is clear from fig. $2 \mathrm{~b}$ that the interaction is much more effective in the presence of the disorder $(W \neq 0)$; while on fig.1b $\mathrm{V}$ had almost no effect, in fig.2b, for a relatively weak disorder, $\mathrm{V}$ leads to a significant decrease of the current. Such a striking influence of the interaction V is also seen in the metallic regime at half-filling (compare e.g. $V=0$ and 1 in figs.1a and $2 a)$.

At this point, we would like to describe more qualitatively the transition from the localized regime (insulator) to the ballistic one (perfect metal). As stressed by Scalapino and al. [12, the Drude weight $\pi D$ is a relevant parameter to characterize both of them. As originally noted by Kohn [13], the Drude weight $\pi D$ can be calculated from the dependence of the ground state energy versus $\Phi$,

$$
D=\frac{N}{4 \pi^{2}}\left(\frac{\partial^{2} E(\Phi)}{\partial \Phi^{2}}\right)_{\Phi=\Phi_{m}}
$$

where $\Phi_{m}=0$ or $1 / 2$ is the location of the minimum of $E(\Phi)$. As mentionned earlier, $\Phi_{m}$ depends on the parity of the number of electrons. For an even electron number parity, we take $\mathrm{D}$ as the second derivative at $\Phi_{m}=1 / 2$.

Note that for free electrons $D=<n>/ m$ where $\langle n>$ is the density of the mobile charge carriers and $m=1 / 2 t$ is their mass. Generally D is given by [14] 


$$
D=\frac{<n>}{m^{*}}
$$

where $m^{*}$ is the effective mass of the carriers renormalized by the interaction. By this way, the D parameter can determine the different regimes. A perfect metal (ballistic regime) will be caracterized by a finite value of $\mathrm{D}$. This corresponds to a persistent current $I$ scaling as $1 / N$. In the insulator, $\mathrm{D}$ vanishes exponentially as the size of the system goes to infinity, $D \propto e^{-N / \xi}$, where $\xi$ is the localization length. As a check of the numerical calculations on the drude peak, we observed the correct finite size scaling for $W=0$, at half-filling $D \sim D_{\text {lim }}+a / N^{2}$ for $V<1$ and $D \propto e^{-N / \xi}$ for $V>1$ when $N>\xi$.

Since we consider disordered systems, we have to average over many realizations of the disorder. The number of configurations we averaged over vary from 50 to 250, depending on the size of the Hilbert space and the filling. In fig.3 $(<n>=0.5)$ and fig.4 $(<n>=0.25)$ D is plotted versus $1 / \mathrm{N}$ ( $\mathrm{N}$ is the size of the ring). We first consider the half-filled case (fig.3). When $\mathrm{V}=0$ and $\mathrm{W}=0, \mathrm{D}$ goes to a finite value in the thermodynamic limit $\left(D_{\text {lim }}=1 / \pi\right)$. As long as $\mathrm{W}=0$ (i.e. without disorder), the Drude weight is weakly affected by a small interaction $V<1$, signature that the system remains metallic. But for $V>1$ (here $\mathrm{V}=2$ ) D decreases faster with $\mathrm{N}$, the system becomes an insulator. We now turn to the effect of disorder. Once $W \neq 0$, we observe a tendancy towards localization for all $\mathrm{V}$. With disorder in the system, the effect of the interaction is also to increase the degree of localization. In the light of the results given by fig. 3 and 4, it appears that the role played by the interaction in presence of disorder $(W \neq 0)$ is clearly different for $\langle n\rangle=0.5$ and for $\langle n\rangle=0.25$. On one hand, at $\langle n\rangle=0.5$ we see that the interaction $\mathrm{V}$ leads to a real decrease. For example, if we consider in fig. 3 the case $W=2$ and compare the data for $V=0$ and 2 we notice that the presence of the interaction reduces the localization length $\xi$ by more than a factor 3. On the other hand, at $\langle n\rangle=0.25$ (and fixed $\mathrm{W}$ ) the value of $\mathrm{D}$ is less affected by the interaction $\mathrm{V}$, as also observed on figs.2a and $2 \mathrm{~b}$ for the current. However, we note that the effect of $\mathrm{V}$ is significantly larger for $W \neq 0$ than in the pure case $W=0$. In summary, we do not observe any increase of $\mathrm{D}$ due to the competition between the interaction and the disorder.

We finish this paper by a few remarks on the conductivity spectrum. The optical con- 
ductivity is given by ,

$$
\sigma(\omega)=\pi D \delta(\omega)+\sigma_{r e g}(\omega)
$$

where $\sigma_{r e g}(\omega)$ given by the Kubo formula

$$
\sigma_{r e g}(\omega)=\frac{\pi}{N} \sum_{m \neq 0} \frac{|<m| \hat{j}|0>|^{2}}{E_{m}-E_{0}} \delta\left(\omega-\left(E_{m}-E_{0}\right)\right)
$$

and $\hat{j}$ is the current density operator

$$
\hat{j}=-i t / 2 \sum_{\mathbf{i}}\left(c_{\mathbf{i}}^{\dagger} c_{\mathbf{i}+\mathbf{1}} \exp (2 i \pi \Phi / N)-h c\right)
$$

All quantities in (9) are calculated at $\Phi=\Phi_{m}$ and $E_{n}$ are the excited manybody energies. The amplitude D of the Drude $\delta(\omega)$ peak was calculated previously. We have explicitely checked the sum rule,

$$
\int_{0}^{\infty} \sigma(\omega) d \omega=-(\pi / 2 N)<0\left|H_{k i n}\right| 0>
$$

where $<0\left|H_{k i n}\right| 0>$ is the groundstate expectation value of the kinetic energy.

In fig.5, $\sigma(\omega)$ is plotted versus $\omega$ for a 16 site ring at half-filling, with $V=0.5$ and for $W=0$ or $W=1$. In presence of the disorder we had averaged over 100 configurations. We clearly see, as expected, that in the metallic case $W=0$, the contribution at non zero frequency is negligeable $(\pi D \sim 0.98$ and only less than $1 \%$ of the weight is left at finite frequencies). However, when we introduce disorder, a strong absorption appears at non zero frequency with a peak around $\omega=0.4 t$, while weight is removed from $\omega=0(\pi D \sim 0.52)$.

This broad absorption can be interpreted physically in the following way: if we assume that disorder localizes the wavefunctions in small finite size regions with a broad distribution of volumes, this leads to a correspondingly broad distribution of finite characteristic frequencies. In other words, the localized electrons can oscillate in disconnected regions of different sizes.

Let us summarize the main result of this paper. In our model for interacting electrons, we never observe an increase of the persistent current when interaction is switched on. At halffilling, the interaction induces a metal-insulator transition . The current strongly decreases, in qualitative agreement with the result of ref. [11]. Away from half-filling, the effect of the 
interaction is much weaker in the absence of disorder. However when impurity scattering exists, the interaction plays again a crucial role and leads to an additional decrease of the current. This is because it is more difficult to move correlated electrons in a random potential than independent electrons. Our results are in discrepancy with those of [7]. It will be of interest to know if the qualitative results obtained in this paper still apply for a multichannel ring.

Support from the Centre de Calcul Vectoriel pour la Recherche (CCVR), Palaiseau, France is acknowledged. Laboratoire de Physique Quantique (Toulouse) and Laboratoire de Physique des Solides (Orsay) are Unités Associées No. URA505 and URA2 du CNRS respectively. 


\section{REFERENCES}

* $\quad$ Electronic address: georges@siberia.ups-tlse.fr

** Electronic address: didier@occitana.ups-tlse.fr

$\dagger$ permanent address: Laboratoire de Physique des solides, Université Paris-Sud, 91405 Orsay, France. Electronic address: gilles@vayu.ups.circe.fr

[1] V. Ambegaokar and U. Eckern, Phys. Rev. Lett. 65, 381 (1990).

[2] L.P. Levy, G. Dolan, J. Dunsmuir and H. Bouchiat, Phys. Rev. Lett. 64, 2074 (1990).

[3] H. Bouchiat and G. Montambaux, J. Phys. (Paris) 50, 2695 (1989); B. Altshuler, Y. Gefen and Y. Imry, Phys. Rev. Lett. 66, 88 (1991); F. von Oppen and E. Riedel, Phys. Rev. Lett. 66, 84 (1991); A. Schmid, Phys. Rev. Lett. 66, 80 (1991).

[4] V. Chandrasekhar, R.A. Webb, M.J. Brady, M.B. Ketchen, W.J. Galager and A. Kleinsasser, Phys. Rev. Lett. 67, 3578 (1991).

[5] R.A. Smith and V. Ambegaokar, Europhys. Lett. 20, 161 (1992).

[6] D. Mailly, C. Chapelier and A. Benoit, Phys. Rev. Lett. 70, 2020 (1993).

[7] A. Muller-Groeling, H.A. Weidenmuller and C.H. Lewenkopf, Europhys. Lett. 22, 193 (1993).

[8] For a recent review see e.g. R. Shankar, Int. J. of Mod. Phys. 15, 2371 (1990).

[9] R.Haydock, V.Heine and M.J. Kelly J. Phys. C 8, 2591 (1975). E.R. Gagliano and A. Balseiro Phys. Rev. lett. 59, 299 (1987).

[10] N. Byers and C.N. Yang, Phys. Rev. lett. 7, 46 (1986).

[11] M. Abraham and R. Berkovits, Phys. Rev. Lett. 70, 1509 (1993).

[12] D.J. Scalapino, R.M. Fye, M.J. Martins, J. Wagner and W. Hanke, Phys. Rev. B 44, 6909 (1991).

[13] W. Kohn Phys. Rev. 133, A 171 (1964). 
[14] D.J. Scalapino, Phys. Rev. B 47, 7995 (1993). 


\section{FIGURE CAPTIONS}

\section{Figure 1}

Current $I(\Phi)$ versus $\Phi$ for a 16 site ring at $\langle n\rangle=0.5$ (fig.1a) or at $\langle n\rangle=0.25$ (fig.1b), fixed $V=0$ and $W=0,0.5$ and 1 .

\section{Figure 2}

Current $I(\Phi)$ versus $\Phi$ for a 16 site ring at $\langle n\rangle=0.5$ (fig.2a) or $<n>=0.25$ (fig.2b), at fixed $W=0$ and $V=0,1$ and 2 .

\section{Figure 3}

Scaling of $\mathrm{D}$ at half-filling $(<n>=0.5)$. D vs $1 / \mathrm{N}$ for different $\mathrm{V}$ and $\mathrm{W}$.

\section{Figure 4}

Scaling of $\mathrm{D}$ at quarter filling $(<n>=0.25)$.

\section{Figure 5}

Total conductivity $\sigma(\omega)$ for a 16 site ring at $<n>=0.5$ and (a) with or (b) without disorder

$(W=0$ and $W=1)$ for a fixed $V=0.5$. For $W=0 \pi D \sim 0.98$. For $W=1, \pi D_{\text {aver. }} \sim .52$ and we averaged over 100 realisations of the disorder. 